Rev. Elev. Méd. vét. Poys trop., 1964, 17, 4 (607-14)

\title{
Étude histochimique du contenu caecal des schistosomes
}

\author{
par P. PICART et S. GRETILLAT
}

\begin{abstract}
RÉSUMÉ
A l'aide d'une série de tests histochimiques différentiels, faits sur coupes histologiques transversales de schistosomes adultes mâles et femeiles (Schistosoma curossoni), il est possıble d'établir l'analogie entre le pigment intracaecal de ces helminthes et celui présent dans le système réticulo-endothélial du foie, des poumons, et des reıns de ruminants infestés par ces parasites.

Ce pigment n'existe que dans les caecums de la femelle, ne contient pas de fer aisément décelable et appartient au groupe des mélanines.
\end{abstract}

Sur schistosomes vivants, examinés immédiatement après prélèvement, il est remarquable de pouvoir suivre, principalement chez la femelle, le trajet des tubes caecaux qui se détachent nettement sur le fond gris blanchâtre du corps de l'helminthe.

Un examen plus détaillé montre que la lumière des caecums est occupée par une masse de couleur marron très foncé chez la femelle et marron plus ou moins clair chez le mâle.

La fixation à l'alcool à $60 / 70^{\circ}$ atténue la couleur et parfois décolore complètement le contenu intestinal du mâle, celui de la femelle gardant sa couleur initiale très foncée.

Efudiant la localisation et la nature du pigment bilharzien présent dans le foie d'animaux de laboratoire infestés expérımentalement par Schistosomo mansoni, Sch. japonicum et Sch. hoematobium, MELENEY et coll. écrivent en 1953 :

«Un pigment hématique, excrèté de l'intestın des vers adultes, s'accumule dans les cellules de Kupffer et les histiocytes des espaces porte et des lésions dues aux œufs,... »
Ces auteurs émettent donc l'hypothèse de l'identité du pigment bilharzien intrahépatique ef du contenu des caecums de I'helminthe.

Nous avons récemmenł signalé (GRETILLAT \& PICART, 1964) chez les femelles gravides de Sch. curassoni, la présence dans le tube intestinal d'un pigment sinon identique du moins très voisin de celui rencontré dans les cellules de Kupffer ef les histiocytes périalvéolaires du poumon de petits ruminants fortement infestés de schistosomiase intestinale.

La présente note est le compte rendu d'un travail fait sur coupes histologiques de schistosomes adultes mâles et femelles, soumises à des tests histochımiques dans le but de déterminer la nature chimique du contenu intestinal des schistosomes.

Matériel d'expérience. - Une trentaine d'exemplaires adultes mâles et femelles prélevés dans les veines mésentériques d'un mouton sacrifié aux abattoirs de Dakar.

Protocole expérimental. - Le matériel est déshydraté, inclus dans la paraffine, puis monté 
sur lames en coupes transversales de $5 \mu$ d'épcisseur.

Une fols déparaffinées, les coupes sont soumises aux tests différentiels suivants, complétés pour certains d'entre eux par une coloration de fond à l'hématoxyline-éosine.

$1^{\circ}$ Fluorescence en lumière de WOOD (lumière ultraviolette).

$2^{\circ}$ Solubilité dans les acides minéraux : $\mathrm{SO}^{4} \mathrm{H}^{2}$ d̀ $10 \mathrm{p} \cdot 100$.

30 Solubulité dans les bases minérales : $\mathrm{KOH}$ à 10 p. 100.

4o Décoloration par les agents oxydants, bain oxydant de MAYER (in Lison, 1960).

$5^{\circ}$ Présence de fer alsément décelable, réaction de PERLS, variante Lison (in Lison, 1960).

$6^{\circ}$ Présence de fer après démasquage, démasquage à l'alcool acide de Macallum (1895 ef 1905).

$7^{\circ}$ Recherche du fer après incinération : a) microincinération de POLICART (1953); b) ef technique personnelle de microlncinération (*).

80 Coloration par colorants des graisses (chromolipordes).

9o Réaction argentaffine par le procédé de MASSON (MASSON, 1914).

100 Solubilité dans les palysulfures, sulfure d'ammonium (in Lison, 1960).

\section{Interprétation des résultats obtenus au cours de ces réactions différentielles}

\section{a) Localisation du pigment infracoecal des schis- tosomes.}

Trouvé en abondance dans la totalıté du tube

(*) Prahquer la microincinération sur un brûleur électrique de HOFFMAN sans aller jusqu'à une minéralisation totale qui détruirait le fin réticule de matières organiques maintenant le pigment en place dans la lumière du cæcum et qui, après incinération va fixer les cendres.

Placer sur la coupe ainsi semi-minéralisée une goutle d'un mélange d̀ parlies égales de :

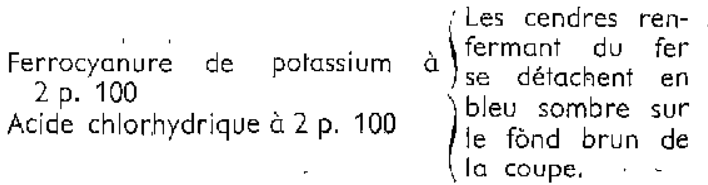

intestinal de la femelle, Il est absent de celui du mâle (photo no 1) (photo $n^{0} 2$ ).

Au niveau du caecum unique (zone des glandes vitellogènes), il est maintenu en place dans la lumière du tube par un fin réticule tendu entre les parais caecales, recouvertes et imprégnées par endroits d'îlots pigmèntaires (photo no 1).

Le pigment est constitué par de petites granulations de 0,6 à $0,9 \mu$ de diamètre, marron foncé et plus ou moins réfringentes. Examiné sous une certaine épaisseur, l'ensemble de la masse centrale, emplissant la lumière intestinale, a une couleur pouvant aller jusqu'au marron très foncé.

b) Résultats des réactions différentielles faites sur coupes histologiques.

Le contenu caecal des femelles:

$1^{0}$ ne présente aucune fluorescence en lumière Wood ;

$2^{\circ}$ est insoluble dans les acıdes minéraux. II esł cependant décoloré par l'alcool sulfurique à 2 p. 100 (alcool à 950). S'agit-il icl dé la réaction utilisée par SAWADA et coll. en 1956, sous le nom de «alcool sulfate »?

$3^{\circ}$ est soluble dans les bases minérales. Se décolore en $15 \mathrm{mn}$ environ dans une solution de $\mathrm{KOH}$ à $5 \mathrm{p} .100$. Seul le réticule maintenant le pigment au centre du caecum persiste si l'on prend certaines précautions (photo $n^{0} 3$ ):

$4^{\circ}$ est décoloré très rapidement par les agents oxydants. Réaction de MAYER au chlore. Comme dans la réaction avec les bases minérales, seul un fin stroma est visible dans la lumière caecale (photo no 4) :

$5^{\circ}$ ne contient pas de fer assément décelable (réaction de PERLS, variante Lison);

$6^{0}$ ne contient pas de fer colorable après technique de démasquage de Macallum à l'alcool acide.

70 après microincinération et imprégnation des cendres par le ferrocyanure de potassium à 2 p. 100 en milieu chlorhydrique, donne les réactions du fer ferrique. Le contenu caecal du mâle donne les mêmes réactions quoique ne renfermant pas de pigment ;

80 n'est pas coloré pạ les colorants des graisses ;

90 réduit les sels d'argentamine (MASSON). II est cependant difficile d'apprécier l'ıntensité 
de la réaction étant donné la coloration initiale brun-noir du substrat (photo no 5);

$10^{\circ}$, n'est pas soluble dans le sulfure'd'ammonium.

\section{DISCUSSION}

A la lecture des résultats rassemblés dans le tableau ci-dessus, le pigment intracaecal des femeiles de schistosomes peut être considéré comme:

10 différent des chromolipoides (absence de fer décelable et non colorable par les colorants des graisses) ;

$2^{0}$ différent de l'hématoïdine qui $n$ 'est pas décolorée par les agents oxydants;

$3^{0}$ différent de l'hémosidérine, qui est soluble dans les acides mınéraux mais non dans les bases, n'est pas décolorée par les agents oxydants et ne donne pas de réaction argentaffine ;

$4^{0}$ différent du pigment type palustre soluble dans le sulfure d'ammonium et qui a une réaction argentaffine négative ;

$5^{\circ}$ analogue aux mélanines par ses caractères de solubilité.

Il est soluble dans l'alcool sulfurique, mais nous n'avons trouvé dans la bibliographie aucune référence quant au comportement des mélanines dans un tel milieu.

Les caecums des femelleś et des mâles de schistosomes renferment du fer que l'an ne peut mettre en évidence que par des techniques brutales de démasquage (microincinération de POLICART). $\mathrm{Ce}$ fer provient vrassemblablement de l'hémoglobine du sang dont se nourrit le schistosome.

Au contraire, le pigment qui n'existe que dans les caecums de la femelle ne contient aucun fer aisément décelable et a des caractères chımiques semblables aux dépôts mélaniques rencontrés dans les cellules du système réticulo-endothélial du foie, des poumons et des reins des animaux fortement infestés de bilharzıose (GRETILLAT et PICART, 1964) (photo no 6).

LEROUX en 1929, signale l'importance des dépôts pigmentaires dans le fore et les poumons d'ovins parasités par Sch. matthei, mais ne donne aucune précision sur leur nature et leur origine.

LAGRANGE et SCHEEMANS en 1951 prétendent qu'ils sont une des résultantes de la digestion du sang par les schistosomes. « L'abondance de pigment résidu de la digestion de l'hémoglobıne, témoigne néanmoins de la consommation du sang $》$.

Malheureusement, ces auteurs n'apportent aucune preuve permettant de rattacher ces déchets à de l'hémosidérine ou à tout autre produit de dégradation de l'hémoglobine.

MELENEY ef coll. en 1953, considèrent le pigment intrahépatique comme identique à celui trouvé dans la fumıère intestinale des schistosomes. lis l'appellent «hematin pigment» sans démontrer cependant qu'il renferme du fer aisément décelable.

COELHO en 1952, met seulement l'accent sur l'action délabrante possible de ceș dépôts intrahépatiques sans parler de leur orıgine et de leur nature chimique.

A notre connaissance, seuls SADAWA et coll. en 1956, par une série de tests analogues à ceux que nous avons utilisés (*), démontrent l'absence de fer et la nature mélanique du pigment bilharzien trouvé 'dans les, cellules de KUPFFER.

N'ayant travaiłlé que sur coupes d'organes (foie), ces auteurs ne pouvaient remarquer la sımilitude chimique existant entre le contenu intracaecal de la femelle du schistosome et les dépôts pigmentares intrahépatiques.

\section{Résumé et conclusion}

A l'aide d'une série de tests histochimiques différentiels, faits sur coupes histologiques transversales de schistosomes adultes mâles et femelles (Schistosoma curassoni), il est possible d'établır l'analogie entre le pigment intracaecal de ces helminthes et celui présent dans le système réticulo-endothélıal du foie, des poumons, ef des reins de ruminants infestés par ces parasites.

Ce pigment n'existe que dans les caca de la femelle, ne contient pas de fer aisément décelable ef appartient au groupe des mélanines.

Instirut d'Elevage et de Médecine vétérinaire des

Pays tropicaux.

Loboratore notional de l'Elevage et de Recherches vetérinaıres.

Services de Brochimie et d'Helminthologie Dokar-Hann (Sénégal)

(*) Pour le dérail de ces techniques, voir l'artıcle GRÉTILLAT \& PICART, 1964, I7, 3, p. 433-40. 


\begin{tabular}{|c|c|c|c|c|c|c|c|c|}
\hline & Chromolipoīde & Hématoídzne & Hémosidérine & Hémoglobine & $\begin{array}{l}\text { Pigment type } \\
\text { palustre }\end{array}$ & Mélenine & $\begin{array}{l}\text { Pigment du foie } \\
\text { poumon, rein }\end{array}$ & Coecum \\
\hline Fluorescence & + & & & & & & & \\
\hline $\begin{array}{l}\text { Solubilitié/ } \\
\text { acide minéral }\end{array}$ & & . & + & & & & & \\
\hline $\begin{array}{l}\text { Solubilitté/ } \\
\text { base minérale }\end{array}$ & & $\div$ & & & $H$ & + & + & + \\
\hline $\begin{array}{l}\text { Décoloration/ } \\
\text { oxydant }\end{array}$ & & & & & + & ++ & + & ++ \\
\hline $\begin{array}{l}\text { Per facilement } \\
\text { décelable }\end{array}$ & + & & + & & & & - & \\
\hline $\begin{array}{l}\text { Fer après } \\
\text { démagquage }\end{array}$ & + & & + & & ++ & & & \\
\hline $\begin{array}{l}\text { Fer après } \\
\text { mícroincinération }\end{array}$ & + & & ++ & 4 & H & & & ${ }^{H}(x)$ \\
\hline Col. des graisses & + & & & & . & - & & \\
\hline $\begin{array}{l}\text { Réaction } \\
\text { argentaffine }\end{array}$ & & + & & & & ++ & + & ${ }^{+}(x x)$ \\
\hline $\begin{array}{l}\text { Solubilité de } \\
\text { Sulfure } \mathbb{N A}^{4}\end{array}$ & & - & & & + & & & \\
\hline
\end{tabular}

(x) - Le fer mis en évidence dens le coecum du mâle et de la femelle n'est décelable qu'après une technique brutale de démasquage (microincinération) Il provient vraisemblablement de l'hémoglobine du seng contenu dans les coeca, et non du pigment lui-même qui, s'il était ferríque aurait sôn fer aisément décelable par les réactiona de Peris ou de Macallum.

(xx) - Une croix unique g'explique par le fait qu'il est parfois dịfficille d'évaluer l'jntensité de la réaction, la coloration initiale brune foncée ḍu substrat gênant la leçture 


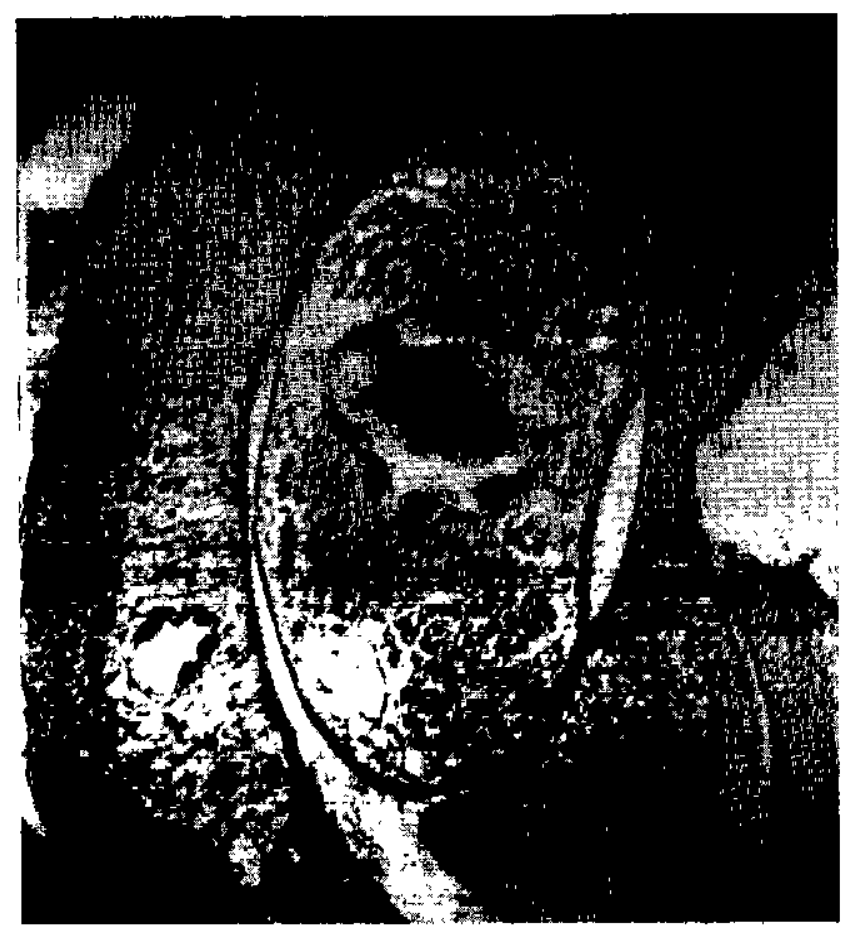

Phota $n^{0} 1$ - Coupe transversale de schistosomes mâle et femelle colorée d̀ I'hématoxylıne éosine. Le caecum unique de la femelle au niveau de la région des glandes vitellogènes contient du pigment qui recouvre les parois du tube intestinal et qui par endroits est inclus dans les cellules mêmes du caecum. Les diverticules caecaux du mâle ne renferment aucun pigment.

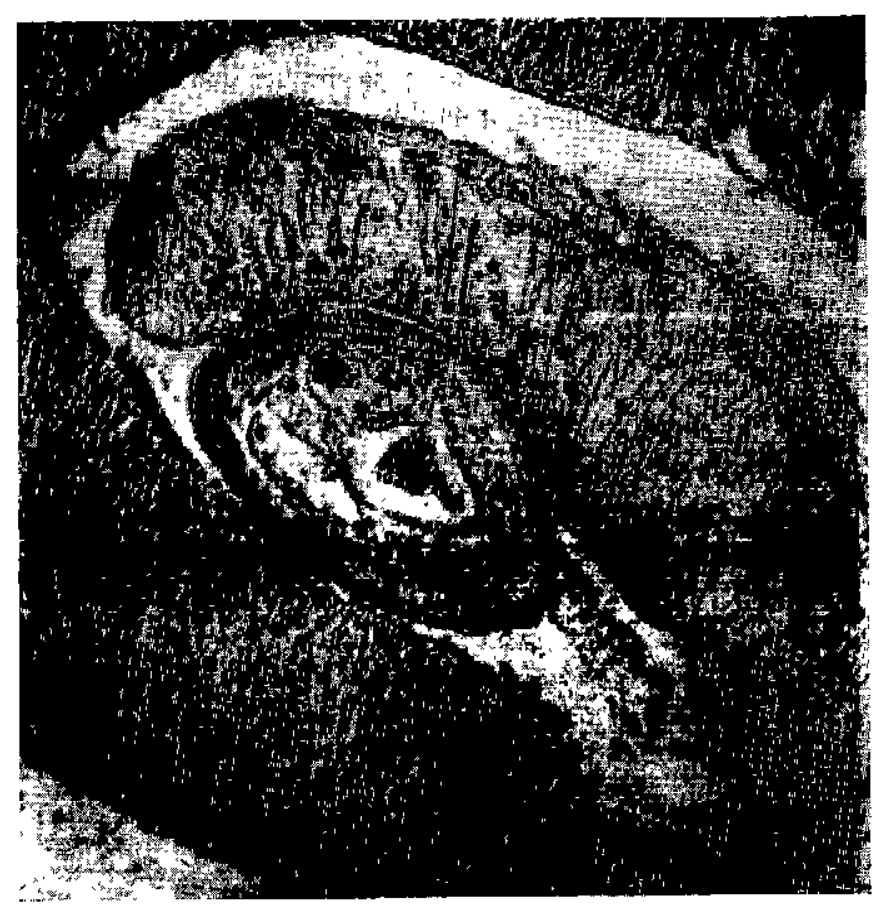

Photo $\pi^{0} 2$. - Coupe transversale de schistosomes mâle et femelle colorée à l'hématoxyline éosine. La coupe passe par la partie antérıeure de la femelle et l'on aperçoit particulièrement dans l'une des branches caecales des amas imporiants de pigment. 


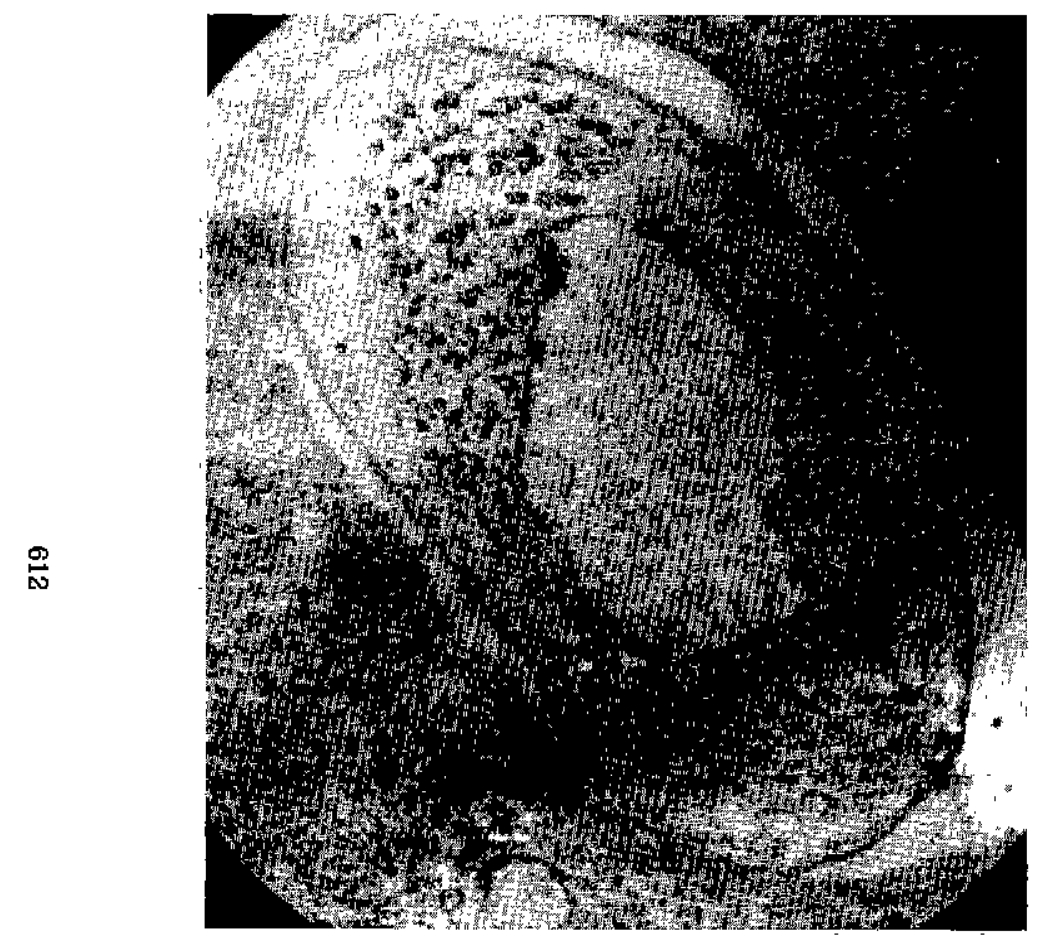

Photo $n^{0} 3$. - Coupe transversale de schislosomes mâle et 'femelle. Traitement par la potasse à 10 p. 100 puis caloration du fond à :l'hématoxyline éosine. Le pigment intracaecal de ta femelle a ëté dissous et il ne reste plus qque "le rétrcule lui servant de support.

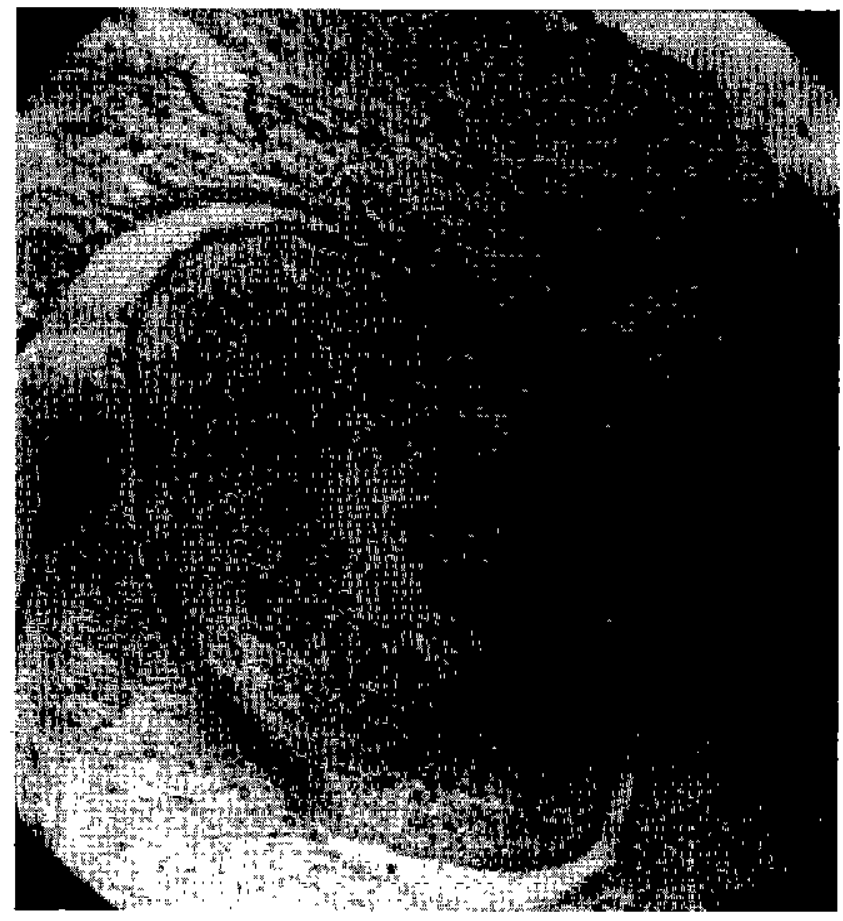

Photo $n^{0}$ 4. - Coupe transversale de schistosomes mâle et femelle. Traitement par les agents oxydants, puis coloration du fond à l'hématoxyline éosine. Le pigment intracaecal de la femelle a été complèfement décoloré et seul n'est visible que le fin réticule qui maintenait en place le pigment. Les glandes vitellogènes sont bien mises en évidence par la coloration finale. 


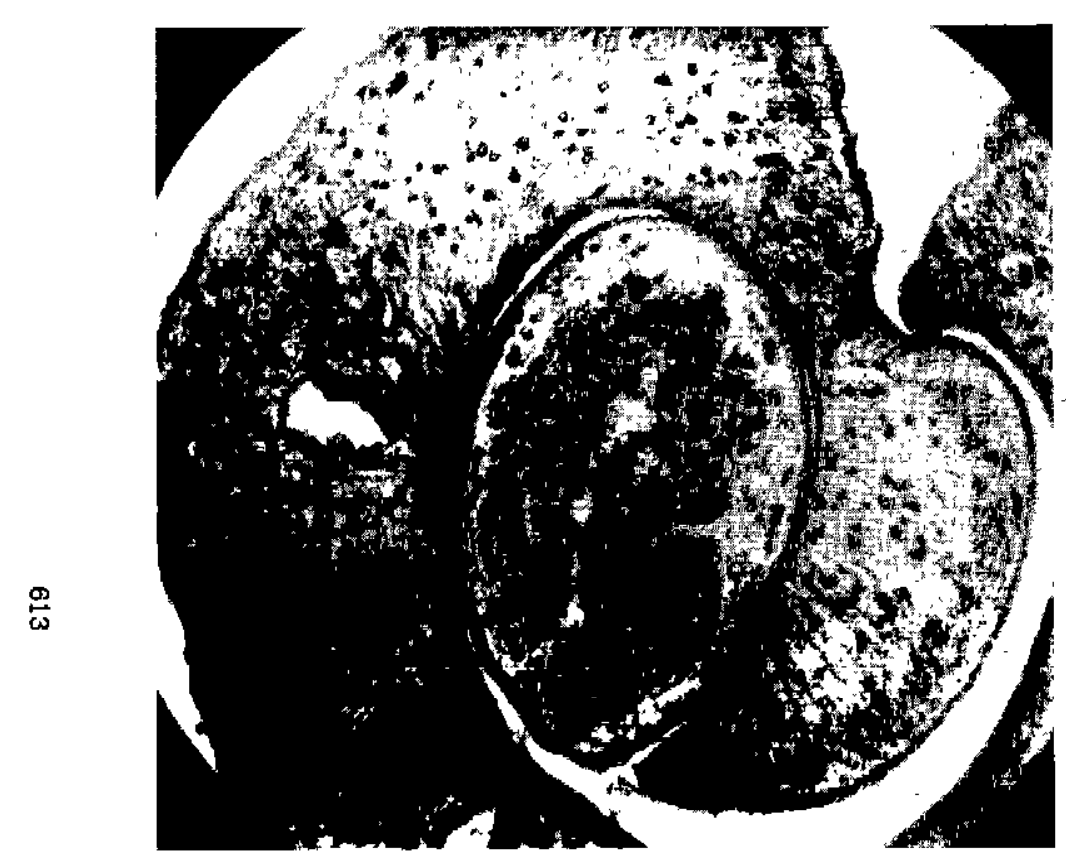

Pholo $n^{n}$ 5. - Coupe Iransversale de schistosomes mâle ef femelle. Soumis à la réaction argentaffine, le pigment caecal femelie apparait très foncé mas le degré d'intensilé de la réaction est difficile à évaluer en raison de la coloralion initiale déjò très sombre du substrat. Les glandes vitellogènes onl aussi une réaction argentaffıne positive, mais certains lests différentiels hislochimiques dont nous ne doninons pas les délails dans la présente note, laissent supposèr que les granulatıons des glandes virellogènes qui se colorent fortement en noir par le procédé de Masson, sont constituées par des polyphénols.

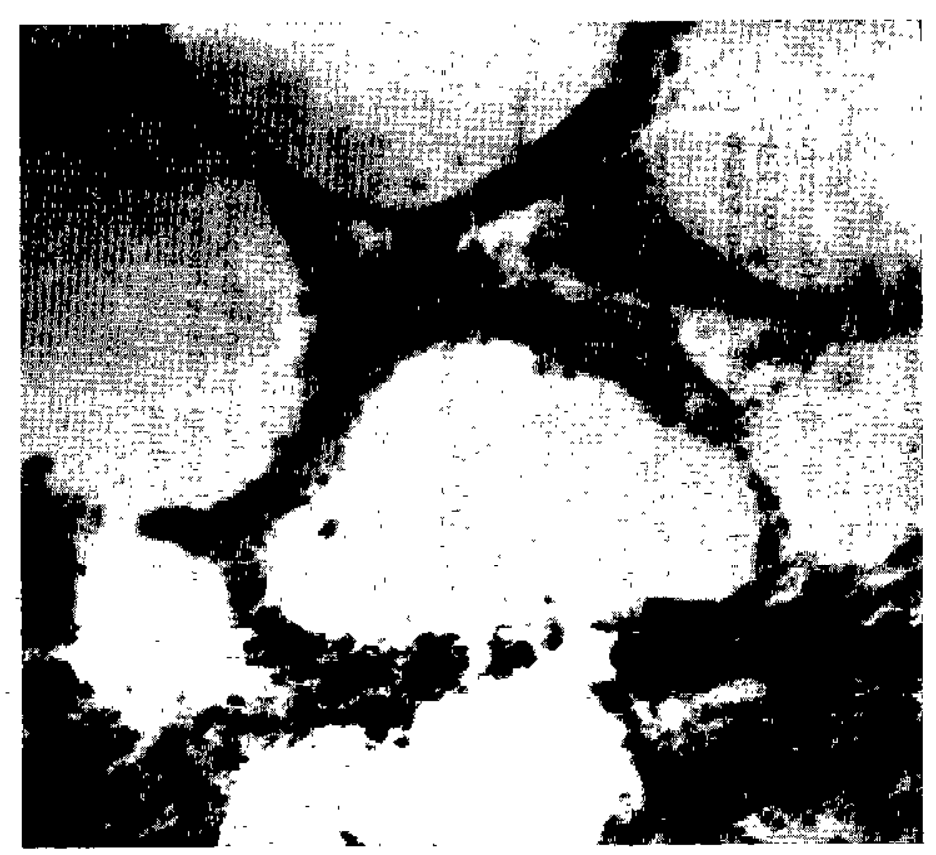

Photo $n^{0}$ 6. - Coupe de poumon d'ovin atteint de schistosomiase intestinale. Coloration à l'hématoxylıne éosinè. Des dépôts massifs de pigment bılharzıen, de nature chimique semblable à celle du pigment intracaecal des femelles de schistosomes sonł localısés au nıveau des histiocyles périalvéolaires pulmonares. 


\section{SUMMARY}

\section{Histochemical sfudy of the cecal contents of the schistosomes}

By means of a series of differential histochemical tests carried out on transverse histological sections of adult male and female schistosomas (Schistosoma curassoni), it is passible to establish the analogy between the intracecal pigment of these helminths and that found present in the reliculo-endothelial system of the liver, lungs and kidneys of ruminants infested by these parasites.

This pigment is only in the female caecum, does not contain easily detectable iron and belongs to the melanin group.

\section{RESUMEN}

Estudio histoquimico del contenido cecal de los esquistosomas

Con una serie de pruebas histoquimicas diferenciales, hechas sobre cortes histológicas transversales de esquistosomas adultos machos y hembras (Schistosoma curassani), es posible establecer la analogía enfre el pigmento intracecal de estos helmintos y el presente en el sistema reticulo-endotelial del higado, de los pulmones, y de los rinones de rumiantes infectados con estos parásitos.

Este pigmento existe solo en el ceco de la hembra, no contiene hierro revelable facilmente $y$ pertenece al grupo de las melaninas.

\section{BIBLIOGRAPHIE}

COELHO, B. (1952). - Publicaçoes Avulsos do Instituto Aggeu Mogolahes, i, p. 61.

GÖNNERT, R. (1955). - Z. Tropenmed. Parasit., 6, p. 257.

GRETILLAT, S. \& PICART, P. (1964), -- Rev. El. Med. vet. Pays trop. ler Congrès Inter. Parasit. Rome, sept. 1964, 17, 3, p. 433.

LAGRANGE, E. \& SCHEEMANS, G. (1951). Ann. Porosit. hum. comp., 26 (4), p. 334.

LE ROUX, P. L. (1929). - 15th Ann. Rept. Dir. Vet. Serv., Un. S. Afr., p. 347.

LISON, L. (1960). - Histochimie ef Cytochimie animales. Principes ef méthodes. Gauthier-
Villars Ed., 3e Ed., 2 vol., 842 pages. MACALLUM, A. B. (1895). - J. microsc. Soc., p. 175.

MACALLUM, A. B. (1905). - J. Physiol., p. 95.

MASSON, M. P. (1914), - C. R. Acad. Sci., 158, p. 59.

MELENEY, H. E., SANDGROUND, J. H., MOORE D. B., MOST, H. et CARNEY, B. H. (1953). - Amer. J. trop. med. hyg., 2, p. 883.

POLICARD, A. P. (1953). - Bull. hist. Appl., 51. p. 160.

SAWADA, T., HARA, K., TAGAKI, K., NAGAZAWA, Y. et OKA, S. (1956), - Amer, j. trop. med. hyg., 5 p. 847. 